the efficacy of oral iron in treatment was not available. (Bokkala S, Napalinga K, Pinninti N, et al. Correlates of periodic limb movements of sleep in the pediatric population. Pediatr Neurol July 2008;39:33-39). (Respond: Dr Sanjeev V Kothare, Department of Neurology, Children's Hospital Boston, Harvard Medical School, Fegan 9, 300 Longwood Avenue, Boston, MA 02115. E-mail: sanjeev.kothare(achildrens.harvard.edu).

COMMENT. The significance of periodic limb movements of sleep is controversial, some authorities dismissing the movements as a normal variant of arousal patterns, not associated with disturbed sleep and not requiring treatment. (Mahowald MW. Con J Clin Sleep Med 2007;3:15-17). Sir Charles Symonds who first described the entity as nocturnal myoclonus affecting 5 adult patients, regarded the movements as a variant of epilepsy (Symonds CP. J Neurol Neurosurg Psychiatry 1953;16:166-171). EEGs obtained in 3 patients uncovered bioccipital slowing in one but none showed epileptiform discharges.

\title{
TRAUMATIC BRAIN INJURY
}

\section{EXECUTIVE FUNCTIONING AFTER TRAUMATIC BRAIN INJURY}

The Behavior Rating Inventory of Executive Function (BRIEF), a caregiver-report questionnaire, was used to measure changes in executive function in the first year after traumatic brain injury (TBI) in a study of children, aged 5 to 15 years, at University of Minnesota, Minneapolis, and Johns Hopkins University School of Medicine, Baltimore, MD. BRIEF reports were obtained at baseline (retrospectively), and at 3 months and 1 year after injury in 330 children with mild-to-severe TBI and in 103 controls with orthopedic fractures. Children with TBI had more dysfunction than controls on the Global Executive Composite at 3 months after injury, and on the Behavioral Regulation Index, Metacognition Index, and Global Executive Composite at 1 year after injury. Between $18 \%$ and $38 \%$ of children with TBI had significant executive dysfunction in the first year after injury, children with more severe TBI having the greater dysfunction. Only $8 \%$ to $10 \%$ of children with orthopedic fractures showed executive dysfunction in the year after in jury. The working memory scale was the only scale to discriminate between controls and all 3 TBI severity groups at both 3 and 12 months after injury. Predictors of greater Global Executive Composite dysfunction were preexisting learning/behavior problems, lower respondent education, and poor family functioning. More systematic screening for executive dysfunction after TBI is recommended to increase recognition of cognitive disability, especially in patients with preinjury learning and behavior disorders and in dysfunctional families. (Sesma HW, Slomine BS, Ding R, McCarthy ML, and CHAT Study Group. Executive functioning in the first year after pediatric traumatic brain injury. Pediatrics June 2008;121:e1686-e1695). (Respond: Heather Whitney Sesma PhD, University of Minnesota, Department of Pediatrics, Mayo Mail Code 486, 420 Delaware Street SE, Minneapolis, MN 55455. E-mail: hwsesma(a umn.edu).

COMMENT. Executive functioning, or the ability to perform sustained, goaldirected, multiple-step skills while inhibiting inappropriate behavior, is dependent on intact frontal-striatal brain circuits. Executive functioning is impaired following TBI that interrupts these frontal lobe connections; the more severe the injury, the greater the degree of executive dysfunction. The BRIEF questionnaire is more valid than performance-based, distraction-free 
measures of executive function, since the caregiver observes the child during unstructured daily activities. The test requires 10 minutes to administer and may be completed from the physician's office. When reports are suggestive of executive dysfunction, a more extensive neuropsychological evaluation is indicated to define the symptoms of ADHD and signs of cognitive dysfunction, the frequent sequelae of TBI. Several articles connecting symptoms of ADHD and learning disorders with TBI are cited in the literature.

\section{SEIZURE DISORDERS}

\section{CLEFT PALATE IN INFANTS EXPOSED TO LAMOTRIGINE DURING PREGNANCY}

Infants with major malformations born to 791 women who had taken lamotrigine as monotherapy during the first trimester of pregnancy, and had enrolled in the North American AED Pregnancy Registry, were identified in a study at the Genetics and Teratology Unit, MassGeneral Hospital for Children, Boston, and Boston University School of Medicine, MA. Of 684 lamotrigine-exposed infants included, $16(2.3 \%)$ had major malformations identified at birth. Five (7.3/1000) had oral clefts: isolated cleft palate (3), isolated cleft lip (1), and cleft lip and palate (1). In comparison, the prevalence of isolated oral clefts in 206,224 unexposed infants born at Brigham and Women's Hospital, Boston, was 0.7/1000 and one tenth that of exposed infants. Of infants enrolled in 5 other registries, 1,623 were exposed to lamotrigine, as monotherapy, and 4 had oral clefts, a prevalence of $2.5 / 1000$. (Holmes LB, Baldwin EJ, Smith CR, et al. Increased frequency of isolated cleft palate in infants exposed to lamotrigine during pregnancy. Neurology May 27, 2008;70:2152-2158). (Reprints: Dr LB Holmes, Genetics Unit, MassGeneral Hospital for Children, CPZS-504, 175 Cambridge Street, Boston, MA 02114. E-mail: holmes. lewis(ìmgh harvard.edu).

COMMENT. Infants exposed to the anticonvulsant lamotrigine during the first trimester of pregnancy have an increased risk of an isolated cleft palate or cleft lip deformity.

\section{TREATMENT OF REFRACTORY STATUS EPILEPTICUS}

The literature on the management of refractory status epilepticus is reviewed and a treatment algorithm suggested by researchers at Children's Hospital of Philadelphia, PA. The definition of status epilepticus (according to Wasterlain et al, 2006) is divided into an 'impending' or early stage (5-30 min) and an 'established' stage of status epilepticus (30-60 min). Impending status epilepticus is a continuous generalized convulsive seizure for at least $5 \mathrm{~min}$, or continuous non-convulsive seizures or focal seizures for at least $15 \mathrm{~min}$, or two seizures without full recovery of consciousness between them. Established status epilepticus is a continuous seizure for at least $30 \mathrm{~min}$, or intermittent seizures without full recovery of consciousness for $30 \mathrm{~min}$. In refractory status, seizures persist despite treatment with 2 or 3 anticonvulsant medications for $30 \mathrm{~min}, 1 \mathrm{hr}, 2 \mathrm{hrs}$ or longer. Based on the literature review, the recommended protocol for impending status epilepticus (SE), $<5 \mathrm{~min}$, begins with buccal midazolam or rectal diazepam, before arrival at hospital. After $5 \mathrm{~min}$, give lorazepam or 